University of Wollongong

Research Online

Faculty of Social Sciences - Papers (Archive) Faculty of Arts, Social Sciences \& Humanities

2014

The feasibility of telephone follow-up interviews for monitoring treatment outcomes of Australian residential drug and alcohol treatment programs

Frank Deane

University of Wollongong, fdeane@uow.edu.au

Peter James Kelly

University of Wollongong, pkelly@uow.edu.au

Trevor Crowe

University of Wollongong, tcrowe@uow.edu.au

Geoffrey Lyons

University of Wollongong, glyons@uow.edu.au

Elizabeth Kate Cridland

University of Wollongong, ekc977@uowmail.edu.au

Follow this and additional works at: https://ro.uow.edu.au/sspapers

Part of the Education Commons, and the Social and Behavioral Sciences Commons

Research Online is the open access institutional repository for the University of Wollongong. For further information contact the UOW Library: research-pubs@uow.edu.au 


\title{
The feasibility of telephone follow-up interviews for monitoring treatment outcomes of Australian residential drug and alcohol treatment programs
}

\author{
Abstract \\ Background: Telephone follow-up interviewing is one method of monitoring treatment outcomes of \\ individuals involved in drug and alcohol treatment programs. The present study is the first to examine the \\ feasibility and generalizability of data obtained from telephone follow-up interviews after drug and alcohol \\ treatment in Australia. Methods: Participants attended 1 of 8 Salvation Army Recovery Service Centres \\ where staff administered outcome measures at intake. Three-month postdischarge telephone follow-up \\ interviews were conducted by researchers from the Illawarra Institute for Mental Health, University of \\ Wollongong. Results: A sample of 700 clients was obtained for follow-up (582 males; 118 females). A \\ $51 \%$ follow-up rate was achieved at a cost of US\$82 per completed interview. No significant differences in \\ baseline characteristics between responding and nonresponding participants were found. Conclusions: \\ Overall, the telephone methodology was shown to be feasible and relatively inexpensive. However, the \\ introduction of outcome measures at the service level in parallel with follow-up data collection \\ procedures complicated the collection of response data. The burden of introducing outcome measures in \\ residential services may be reduced by utilizing a phased implementation strategy.

\section{Keywords} \\ feasibility, drug, residential, australian, outcomes, treatment, monitoring, interviews, programs, up, alcohol, \\ follow, telephone

\section{Disciplines} \\ Education | Social and Behavioral Sciences

\section{Publication Details} \\ Deane, F. P., Kelly, P. J., Crowe, T., Lyons, G. C.B. \& Cridland, E. K. (2014). The feasibility of telephone follow- \\ up interviews for monitoring treatment outcomes of Australian residential drug and alcohol treatment \\ programs. Substance Abuse, 35 (1), 21-29.
}


RUNNING HEAD: Feasibility of telephone

TITLE: Feasibility of telephone follow-up interviews for monitoring treatment outcomes of Australian residential drug and alcohol treatment programs

AUTHORS: Frank P. Deane (BSc, MSc, Dip Clin Psych, PhD) ${ }^{1}$

\author{
Peter J. Kelly (BSc (Hon), PhD) ${ }^{1}$ \\ Trevor P. Crowe (BSc (Hon), PhD) ${ }^{1}$ \\ Geoffrey C. B. Lyons (BSc (Hon), PhD) ${ }^{1 *}$ \\ Elizabeth K. Cridland (BPsych) ${ }^{1}$
}

This study was funded in part by a research consultancy from The Australian Salvation Army to the first three authors.

${ }^{1}$ Illawarra Institute for Mental Health, University of Wollongong, Wollongong, N.S.W., 2522, Australia

* Corresponding author: Tel +61 24221 4207; fax: +61 4221 5585; email: glyons@uow.com.au 


\begin{abstract}
Telephone follow-up interviewing is one method of monitoring treatment outcomes of individuals involved in drug and alcohol treatment programs. The present study is the first to examine the feasibility and generalizability of data obtained from telephone follow-up interviews after drug and alcohol treatment in Australia. Participants attended one of eight Salvation Army Recovery Service Centres where staff administered outcome measures at intake. Three-month post-discharge telephone follow-up interviews were conducted by researchers from the Illawarra Institute for Mental Health, University of Wollongong. A sample of 700 clients was obtained for follow-up (582 males; 118 females). A 51\% follow-up rate was achieved at a cost of US $\$ 82$ per completed interview. The only significant difference in baseline characteristics between responding and non-responding participants was that higher rates of Anglo-Australians completed follow-up interviews. Overall, the telephone methodology was shown to be feasible and relatively inexpensive. However, the introduction of outcome measures at the service level in parallel with follow-up data collection procedures complicated the collection of response data. The burden of introducing outcome measures in residential services may be reduced by utilising a phased implementation strategy. The results also highlight the need for further research on ethnicity and personality factors in research methodology studies.
\end{abstract}




\section{INTRODUCTION}

It is important for drug and alcohol treatment providers to implement evidence-based practices (EBPs) into their programs $(1,2)$ and the collection of outcome data is often a component of this process (3). However, treatment organisations are often complex hierarchical systems. As a result the implementation of EBPs is also complex; needing to be done in conjunction with national, local and individual level policy and procedures (3-5). The structure, resources and culture of treatment organisations and individual service centres also complicates the implementation of EBPs and the utilisation of routine outcome measures (3, 4). Furthermore, the characteristics inherent in the substance misusing population (e.g. high mobility, homelessness, unstable living arrangements) can threaten the collection of postdischarge outcomes (6-8). Thus, the collection of post-discharge outcome data is recognised as a particular challenge for drug and alcohol treatment programs $(7,9)$. Two factors to be considered when evaluating the methodologies used to collect outcome data include the feasibility of the method and the generalizability of the data obtained (7).

Non-response is a major barrier to the collection of post-treatment outcome data $(7,9$, 10). Thorough and flexible tracking and follow-up procedures can maximise the quantity and generalizability of follow-up data $(8,11)$. This can include: gathering detailed participant information which includes next of kin contact details, providing participants with reminder letters, toll-free numbers and flexible calling times for returning follow-up calls; providing incentives for participation; using researchers external to the treating organisations for follow-up calls; using a mix of telephone, mail and face-to-face interviews; and contacting next of kin, community and health services regarding the status of non-responding clients $(6$, 7, 11). Further research is required to determine the feasibility of these strategies and the impact they have on response rates and data generalizability. 
Response rates are an important metric for determining the feasibility of a follow-up methodology (3) but published response rates vary considerably. Some research has reported response rates as low as $15-21 \%(12,13)$ and others over $80 \%(14-16)$. More commonly though, projects report rates around 50 to 70 percent. Tiet et al. obtained a $67 \%$ response rate by using a mixture of mail and phone based methods (mail only resulted in $40 \%$ response rate) (10). Rosenheck and Seibyle obtained 30\% to $70 \%$ response rates using mixed face-toface and phone interviews (2) and Oudejans et al. obtained a 53\% response rate with phone based follow-ups (7). The variation in rates is complicated by the fact that there can be different ways in which response rates are calculated (9) which in turn influences the perceived feasibility and validity of research methods (17). The post-discharge follow-up period (e.g. 3-month vs. 12-month post-discharge) and the contact method adopted (e.g. telephone, mail, face-to-face interviews) may also influence response rates. Together, these factors make it difficult to make judgements regarding an acceptable response rate. One proposed benchmark, based on a review of previous longitudinal research, suggests that response rates of more than $48 \%$ are acceptable (7). Further work is required to justify such recommendations and to determine whether this proposed value is feasible. One aspect of determining feasibility is the resources and other costs associated with the various methods (10).

A key element of cost relates to the time taken to achieve post-treatment contact. As with response rate the acceptable window of opportunity for collecting follow-up data is not clearly identified. For example, with a 3-month post-discharge follow-up there is no clear guideline on how long or how frequently researchers should continue to attempt to contact participants before their responses are deemed to fall beyond the desired 3-month period. One month after the intended follow-up date has been proposed (7). Similarly, the acceptable cost of collecting follow-up data is also unclear. In part, this may vary depending on how it is 
calculated. It can be estimated via a 'time investment' (calculated from the average number of attempts to complete a follow-up) or 'financial investment' (estimated from primary costs of implementing follow-ups such as employing the research assistants, total value of participant incentives and costs of telephone calls and mail correspondence). The cost of data collection may be lower in telephone follow-up methodologies. For example, the cost of obtaining a $67 \%$ response rate using mailed questionnaires has been estimated as being as high as US\$186 per participant (10), while telephone follow-up methodology has a lower estimated cost at approximately US\$57 per participant with a $65 \%$ to $53 \%$ response rate (7).

Finally, the generalizability of the methodology can be explored by comparing baseline characteristics of responders and non-responders; however, there is little consensus in the literature as to what characteristics predict follow-up response. Rosenheck and Seibyl found that participants who completed follow-up interviews were younger, more likely to be non-white, have lower incomes, more serious substance use problems and longer treatment periods (2). Tiet et al. found that participants who completed follow-up interviews had significantly lower Addiction Severity Index (ASI) (18) composite scores at intake for drug use and employment but significantly higher ASI psychological and medical composites at intake (10). Oudejans et al. found lower follow-up response rates amongst participants who were polysubstance abusers, seeking treatment for a substance other than cocaine, who were younger, and had more employment, education, legal and family/social support problems. Dutch rather than non-Dutch participants were also more likely to respond (7). Though a broad array of possible determinants is presented by these studies results in the field remain inconclusive and at times contradictory. For example, some suggest younger participants are more likely to be responders (2); others suggest older participants may be more likely (10). Similarly, some have found that those with more severe substance misuse problems may be more likely to respond (2); other studies suggest those with less severe misuse are more likely 
(10). The role of ethnicity, employment status, legal problems, family and social support, and physiological and psychological health on likelihood of response also are unclear.

In summary, there is a great deal of variation in follow-up methodologies utilized and a need for further research on these mechanisms. Feasibility of follow-up methodology can be explored by examining response rates, acceptable follow-up data collection periods, and resources utilised. Generalizability can be explored by determining whether the sample collected differs from the total population. Overall, telephone methodology is one of the most frequently used follow-up methodologies (19) though the feasibility of this method and the generalizability of the data it obtains remain variable. There is currently little published on the implementation of outcome data collection methodologies within Australian residential drug and alcohol treatment programs. This is problematic as attitudes towards substance use (20), substance usage rates $(20,21)$, response to substance misuse treatments (22), and participation in longitudinal research $(2,23,24)$ may vary across ethnic groups. Calls have been made for investigators to consider the role of culture in substance abuse research (20, 25) and national differences are likely to broadly reflect aspects of cultural difference. Thus, this study seeks to clarify how feasible telephone follow-up methods are for gathering treatment outcome data for Australian residential drug and alcohol clients. The aim of the current report is to describe and evaluate the feasibility and effectiveness of (1) implementing routine outcome measures within Australian residential drug and alcohol treatment programs, and (2) implementing a 3-month telephone follow-up procedure with the same population.

\section{METHOD}

\section{Participants}

The present study recruited participants from eight Salvation Army drug and alcohol residential rehabilitation centres located along the east coast of Australia. These residential rehabilitation centres provided eight to 10 months of residential drug and alcohol treatment, 
in the form of a modified therapeutic community. The treatment program, known as 'The Bridge Program', utilised a 12-Step treatment approach primarily based on the disease model of addiction. Upon entering the program clients progressed through a group-based treatment process that involved a combination of skills training, psycho-education, 12-step based interventions and individual counselling.

Measures

Demographics and treatment histories

Demographics were adapted from the Brief Treatment Outcome Measure (26) (BTOM). Questions gathered information on participants' age, preferred language, prior drug use and treatment history. This included the types of substance(s) that had been used in the last 12 months, the substance(s) for which they were seeking treatment, and length (years) of substance use problems. In each question the participant could endorse more than one response from a list. Treatment history items included prior treatment sought for their current drug problems, prior access to a residential rehabilitation treatment service, and previous access to self-help groups such as Alcoholics Anonymous (AA). Participants were also asked if they had ever received treatment for a mental health problem and if they had they were asked to specify their diagnosis.

The Addiction Severity Index

The Addiction Severity Index - Fifth Edition (18) (ASI) is a widely used semistructured interview which assesses an individual's health status across seven domains: Medical Status (8 items), Employment/Support Status (21 items), Alcohol Use and Drug Use (54 items), Legal Status (32 items), Family/Social Relationships (38 items) and Psychiatric Status (23 items). Items from each area are assessed in relative to the participant's lifetime and 30 days prior to assessment. Responses in the 30 days prior to assessment generate composite scores (CS) that indicates problem severity across the seven health domains. The 
ASI has been found to be a reliable and valid measure for mentally ill substance misusers (27-32). The complete ASI was administered at both intake and follow-up however, to avoid repetition the 'lifetime' ASI questions were omitted at follow-up administration.

\section{Retrospective mental health and substance use}

Included in the follow-up interview were six Before and After Rehabilitation (BAR) items which assessed participants' perceived problems with alcohol and drugs, gambling and mental health before and after participation in the treatment program. Items were answered on a 5 point Likert scale ranging from 'No problem' (1) to 'Very large problem' (5). There was also an option for 'Unsure'. A sample item is 'To what extent do you feel you had problems with the use of drugs or alcohol prior to entering the program'. These items were included as an overall indicator of self-perceived change from before to after treatment and were only in the follow-up interviews.

\section{Additional measures}

A range of other measures were also administered to participants during treatment and/or at follow-up, including: the Depression, Anxiety and Stress Scale-21 (33); the Drug Taking Confidence Questionnaire (34); the Desires for Alcohol Questionnaire (35); the Recovery Assessment Scale (36); the Life Engagement Test (37); the Mental Health Continuum - Short Form (38); the Client Assessment Summary (39); the Spiritual Belief Scale (40); the Religious Background and Behaviours Scale (41); the Daily Spiritual Experiences Scale (42); the Heartland Forgiveness Scale (43, 44); the Aggression Questionnaire (45).

Procedure

This study was part of a wider collaborative research initiative between the Australian Salvation Army and the Illawarra Institute for Mental Health (iiMH) at the University of Wollongong. One of the aims of the project was to introduce the use of routine client 
assessment and outcome measures into the daily operations of the Salvation Army Recovery Service Centres. It was within this context that the measures of this study were administered. Salvation Army RSC managers and clinical employees (intake workers and case managers) were trained in the administration of the ASI and all outcome measures used in this study by researchers from the Illawarra Institute for Mental Health. The administration of these measures was then incorporated into the Salvation Army Recovery Service Centres' intake protocols. Salvation Army treatment providers explained the aims, procedures and demands of the research to each client entering treatment. Each client was provided with an information and consent form and invited to participate. Clients wishing to participate completed the consent form, the ASI and all other assessment measures during this intake session. Consent also included agreement to be contacted for follow-up data collection procedures after discharge. The completion of the intake assessments took approximately 60 minutes. Data from the assessments were then entered by Salvation Army staff into the Salvation Army's online Service and Mission Information System (SAMIS). This data was downloaded from SAMIS by researchers every six months. Participants not consenting to complete the ASI and outcome measures still received treatment as usual. All research procedures were reviewed and approved by the University of Wollongong Human Research Ethic Committee.

The data downloaded from SAMIS was vast and often pertained to multiple treatment admissions for each consenting participant within the study period. Because of this the following criteria were used to cleanse the data and determine eligibility for follow-up. (1) Participants had to have been in treatment in one of the eight Salvation Army Residential Rehabilitation programs between June 2009 and November 2010 and consented to participate in the study. (2) Participants had to have completed the ASI assessment within 30 days of intake (including having an ASI completion date attached to their intake assessment data). 
This 30 day window was set to allow for possible withdrawal effects experienced by individuals entering treatment and because many of the ASI items were based on experiences in the 30 days prior to assessment. In addition, those without an ASI were not followed-up since baseline and post-discharge ASI data was a key outcome variable. (3) Data must have been from the client's first admission to the treatment program. Any data pertaining to a participant's second or subsequent admission was removed from the study. This was done to minimise the influence of extraneous variables on the recovery process (e.g. participation in additional treatment programs between admissions to Salvation Army services) and thus give a clearer indication of the effectiveness of the Salvation Army's substance abuse programs as a standalone treatment. (4) Participants had to have provided forwarding details for followup.

A three month post-discharge follow-up was set. Follow-up attempts began approximately two weeks prior to the follow-up date and were focused until four weeks post the follow-up date. Follow-up phone calls were conducted from the University of Wollongong. Names of clients discharging from the treatment programs were downloaded from SAMIS on a weekly basis. Follow-up phone calls were made by trained research assistants who were external to the Salvation Army. To increase the chance of contact, follow-up calls were made at varying times and days of the week including outside of business hours. Individuals indicating lack of time to complete the full follow-up were given the option of either scheduling an alternate time for contact or completing only the BAR items, which took approximately two minutes to complete. Participants who completed either the full follow-up interview or the BAR were provided with a gift voucher (AUS\$20) that could be used at several major retail outlets (e.g. grocery stores, department stores, book shops). These gift vouchers could not be used for purchasing alcohol or tobacco products. 
Several protocols were developed to maximise follow-up response rates (Figure 1). Specifically, after four unsuccessful follow-up attempts two procedures were followed. Firstly, the next of kin specified by the participant at intake was contacted and asked to complete a brief 'Next of Kin Assessment'. This assessment was essentially the BAR items reconstructed to assess the next of kin's perception of the participants' problems concerning alcohol and drugs, gambling and mental health before and after the program. The next of kin also had the opportunity to provide current contact information for the participant. Secondly, participants who could not be contacted by phone were sent a reminder letter. This letter was addressed to the participants' nominated postal address and included the brief assessment form a request for current contact details and a preferred date and time for a follow-up phone interview, and self-addressed return envelope.

\section{RESULTS}

Generalizability of the Study: Representativeness of Sample

The total number of data cases downloaded from SAMIS was 2196. Data cleansing removed 1496 cases from the data set. Reasons for this included ASI date issues (e.g. ASI assessment dates not being recorded; the ASI being conducted after 30 days of treatment) ( $n$ =996); ASI not being administered $(n=184)$; client data pertaining to second or subsequent treatment admissions $(n=136)$; client not consenting $(n=124)$; no client contact details recorded $(n=56)$. These ineligible participants had a mean age of $37.27(\mathrm{SD}=10.24), 83 \%$ were male, and there was an average time in treatment of 88 days $(\mathrm{SD}=86.05)$. Primary substances of abuse included alcohol (57\%), amphetamines (16\%), cannabis (14\%), heroin (9\%), and other (4\%). Ethnicity consisted of Anglo-Australian (75\%), Aboriginal and Torres Strait Islanders $(9 \%)$ and other $(16 \%)$. Twenty-seven percent had been educated to a lower secondary level, $60 \%$ to upper secondary, and $11 \%$ had post-secondary education. Twentyeight percent were employed in a full-time capacity prior to treatment, $27 \%$ part-time, $7 \%$ 
unemployed, $15 \%$ in a controlled environment, and $23 \%$ were employed in another capacity (e.g. student, homemaker).

The data cleansing left a final sample of $700(32 \%)$ participants eligible for follow-up. From this sample 108 participants were not contacted for follow-up due to administrative errors, which included participants not being detected for follow-up via the weekly SAMIS checks. Thus, follow-up information was obtained for 474 (68\% of eligible participants). This contact included: participants who withdrew consent when contacted $(n=24)$; participants who completed the full follow-up telephone interview $(n=358)$; participants who completed the brief follow-up interview only $(n=21)$; or contact with next of kin or significant others ( $n=71)$ who in turn either completed a brief interview $(n=34)$ or reported that the particapant was incarcerated $(n=32)$ or deceased $(n=5)$. The follow-up response rate was calculated by dividing the number of participants who completed the follow-up inteview $(n=$ $358)$ by the total eligible sample $(n=700)$, resulting in a response rate of 51 percent. Figure 2 presents a detailed breakdown of the eligible participants. Table 1 presents the demographics and histories of all participants eligible for contact.

Chi square analyses were conducted to explore whether differences existed between contacted and non-contacted participants on demographic and other descriptive variables. No significant differences in employment $\left(\chi^{2}(14)=7.54, p=.91\right)$, education $\left(\chi^{2}(10)=8.09, p=\right.$ $.62)$, gender $\left(\chi^{2}(2)=3.56, p=.17\right)$, marital status $\left(\chi^{2}(10)=9.27, p=.51\right)$, religious identity $\left(\chi^{2}(4)=5.79, p=.21\right)$ and primary substance of misuse $\left(\chi^{2}(8)=7.16, p=.47\right)$ was found between those who were contacted at follow-up (contacted), those who were unable to be contacted (no contact made) and those who were not attempted to be contacted because of administrative errors (not attempted). Ethnicity was shown to significantly differ with more Anglo-Australian participants being contacted than Indigenous or non-Anglo Australians $\left(\chi^{2}\right.$ 
$(2)=7.33, p=.03)$. Table 1 outlines the demographic variables for the sample according to follow-up category.

Differences in clinical characteristics were explored using multivariate analyses of variance (MANOVA). Participants' follow-up status (contacted, not contacted, not attempted) were entered as the independent variable and age, years of problem usage, number of previous treatment attempts and days in treatment were the dependent variables. Violations of normality were corrected using logarithmic and square root transformations. Homogeneity of variance-covariance and multicollinearity assumptions were met. No significant effect of follow-up condition was found on the dependent variables, $F(8,1312)=1.14, p=.18$; Wilks' Lamda $=.98$; partial $\eta^{2}=.009$. A second MANOVA was conducted to determine if participants' intake ASI composite scores (including, medical, psychiatric, family/social, employment, legal, alcohol and drug status) influenced their follow-up status. Assumptions of normality, homogeneity of variance-covariance and multicollinearity were met. No significant effect of ASI composite scores at intake on the follow-up condition was found, $F$ $(14,20)=2.26, p=.053$; Wilks' Lamda $=.13$; partial $\eta^{2}=.64$. Table 2 outlines ASI Composite Scores from the intake assessment according to group.

Feasibility of Study

Days between discharge and follow-up contact ranged from 68 to 217 with a mean of 108 days $(\mathrm{SD}=30.23)$. The upper range ( 217 days $)$ is higher than our 90 -day target because many participants' follow-up interviews were continually rescheduled. This often occurred when a participant indicated a willingness to complete the follow-up interview but repeatedly rescheduled the interview time, or where participants had unstable accommodation resulting in multiple contacts with family members prior to contacting the participant. The lower range (68 days) was slightly below our 90 day target because of participant and administrative 
factors (e.g. avoiding researcher absenteeism during holiday periods; participants requesting an earlier follow-up). Eleven percent of respondents completed their follow-up assessment before the 90-day follow-up period, $60 \%$ within the 90 -day follow-up period, and $29 \%$ after the 90-day follow-up period. Approximately 2240 follow-up phone calls were made between June 2009 and November 2010. The average number of contact attempts per participant was 3.78 with a range of 1 to 22 . In total AUS $\$ 2499$ was spent on line charges for telephone follow-up calls during the study period and approximately AUS $\$ 8200$ was spent on gift vouchers. Using an estimated staff average wage of AUS $\$ 33.58$ per hour and an estimated 90 minutes dedicated to each successful follow-up interview (60 minutes for the interview and 30 minutes for data entry and associated administrative tasks) results in a total estimated cost of AUS\$93.96 per completed follow-up. This equates to approximately \$US82 at the time of the data collection (2009 to 2010) (http://www.oanda.com/currency/average).

\section{DISCUSSION}

This study examined whether the implementation of routine telephone follow-up was feasible for assessing treatment outcomes in Australian residential drug and alcohol treatment centres. A major criteria used to assess the generalizability and feasibility of follow-up research is response rate (7). Dividing the number of completed follow-ups by the number eligible for follow-up resulted in a response rate of 51 percent. This was comparable with previous studies $(2,7,8,10)$ and beyond the proposed acceptable rate of $48 \%$ (7). This percentage should not necessarily be seen as an acceptable goal for future follow-up studies, but rather a realistic and achievable percentage that researchers and service providers should aim to improve through further methodological research.

We obtained post-discharge information (the number of people contacted [including next of kin] divided by the number of participants eligible for follow-up) for $68 \%$ of participants while non-response and refusal rates were $17 \%$ and $6 \%$ respectively. The average 
time between discharge and follow-up was 108 days, which was within the one month window proposed by Oudejans et al. (7). The estimated cost for each completed follow-up interview was US\$82. This cost is slightly higher than the US\$57 found by Oudejans et al. (7) but lower than the US\$186 found by Tiet et al. (10) for mail methodology.

Overall, the results are broadly consistent with prior research (7) and suggest that telephone methodology can produce acceptable response rates while being less expensive than mail methodology. However, it is important to highlight that these follow-up cost estimates do not include the time required by researchers and administrators to establish the follow-up systems. Consultations with organisations, training costs and database development are all examples of one-off set-up costs that will be required of organisations who wish to establish follow-up protocols. Other costs can accrue for sustaining routine outcome assessment, but should eventually become a component of normal operational costs. These include items such as maintenance of information systems and training new staff in measurement administration at intake and any other points where services wish to understand rates of improvement amongst their participants.

Several common barriers were identified during the study. Participants often expressed a lack of time and/or disinterest in the study and completing the outcome measures. Some participants were also sceptical about the anonymity and confidentiality of their responses. We attempted to address these concerns by assigning the same research assistant to each participant when several call attempts occurred $(6,46)$, educating the participants on the purpose of the research and the importance of obtaining positive and negative feedback, assuring them of their confidentiality, and providing them with the options of either rescheduling their follow-up interview to a more convenient time or completing only the short BAR items. In addition to these problems we also had difficulty tracking the clients. This population is very mobile because they often have unstable housing and living arrangements 
which are associated with their addictions $(47,48)$. Several tracking and follow-up strategies were used to address this challenge. For example, we mailed reminder letters and brief surveys to non-responding clients, provided a toll-free 24 hour number (with message bank after hours) for participants to contact us, and contacted nominated next-of-kin regarding the participant's location and status. Other options for future research may include obtaining information about participants' potential future moving plans $(19,46)$, providing participants with project branded stationary (which includes research contact details) which they can use to update their contact details $(8,19)$, and utilising multiple media formats such as the internet and text messaging to remind participants of scheduled follow-ups $(11,49)$.

In addition to these participant level problems several service level problems were encountered. Some of the most common being that the participants' contact details were often not captured in the intake assessments or lacking in detail. Occasionally, contact details were entered incorrectly into the SAMIS database at intake, and intake assessment dates were not recorded. The lack of an assessment date was problematic because it resulted in confusion as to whether (a) the intake assessments and ASI data pertained to a client's first, second or subsequent admission or (b) whether the intake assessment had actually been completed within the specified 30-day post-admission assessment period. These were important eligibility criteria because without them there was no valid baseline data to be able to make pre-treatment and follow-up comparisons. We could have chosen to loosen our criteria by utilizing only follow-up data (without baseline being required) in the assessment of service effectiveness, but this was viewed as less desirable because it would have restricted our ability to investigate differences in pre and post-treatment outcomes. Similarly, these difficulties may also have been partially due to the fact that we followed-up all participants entering treatment, regardless of treatment duration. As a result some participants targeted for follow-up may have been engaged with The Salvation Army treatment services for a very 
brief period of time which also may have restricted proper intake procedures from being followed. However, we felt it was important to try and provided data on all clients entering treatment. Together, these problems resulted in the loss of a large subset of the data $(n=$ 996). Though minimal differences were found between these lost participants, those who were contacted, and not-contacted participants, future research could avoid these problems and increase their overall sample size by reinforcing to case workers the importance of accurate intake assessment dates and data entry procedures.

Apart from exploring the feasibility of the telephone methodology we also assessed the generalizability of the follow-up data obtained by exploring differences on demographic variables across those contacted at follow-up, those unable to be contacted and those eligible but not attempted to be contacted because of administrative errors. In contrast to previous research $(2,7,10)$ participants' characteristics and ASI composite scores at baseline generally did not significantly differ. This suggests that data from those who completed the follow-up is likely to be mainly representative of those entering the programs. It offers some reassurance that outcomes obtained from follow-up may not be significantly biased. However, it should also be noted that the reliability of the ASI composite scores has been questioned (50). Further research is required on both the roles of psychological, physiological and social wellbeing at intake on follow-up response rates and the reliability and validity of the self-report measures used to capture this data in substance abuse populations (51).

Overall, the only difference in our data was that Anglo-Australian participants were more likely to be contacted at follow-up suggesting the need for some caution in generalising to non-Anglo participants. Previous research has found non-Anglo participants to be more likely to complete follow-ups (2). It is possible that there are specific Australian cultural factors at work that explain these differences. For example, Indigenous Australians (Aborigines and Torres Strait Islanders) often have the highest socio-economic disadvantage 
(52). As a result access to stable living arrangements and telephone may be lower, thus reducing the frequency with which they were able to be contacted for follow-up. Previous research shows that ethnicity can have one of the largest influences on follow-up participation (7). Our results also highlight this and suggest that ethnic minority and minority indigenous consumers (e.g. aboriginal Australians, Native Americans) may be less likely to participate in follow-up research than ethnic majority consumers. Researchers and service providers should consider ways in which they can address this disparity when developing evidence-based practice policies, procedures and research methodologies. In addition, as the majority of baseline characteristics did not predict follow-up response research could also explore more intrapersonal psychological variables. For example, broad determinants of age, gender, socio-economic status or substance misuse severity may not be sensitive enough to reliably predict follow-up response. In contrast, evidence suggests that personality traits such as agreeableness and openness to experience can predict research participation (53). Research methodology studies could therefore explore whether factors such as personality, stage of change, attitude towards the service facility or cognitive flexibility predict follow-up response.

There were several limitations to this study, the main one being the relatively large proportion of participants ineligible for involvement. These participants were excluded due to either the quality of their data, administrative errors or due to decisions about the study design. Nevertheless, the large numbers of these participants raises questions about the generalizability of the results. To address this we have presented some basic demographics and treatment histories of these ineligible participants, which are generally comparable to those of the eligible participants (Table 1). However, further research needs to explore data collection methodologies within large multi-site treatment programs in order to develop new strategies and maximise the validity of the data obtained. In addition, the study was also 
limited by the relatively brief follow-up period and the lack of information available regarding the characteristics of the non-consenting participants (though the 6\% rate of nonconsent was relatively low).

The collection of reliable and valid baseline data in treatment programs is acknowledged as a particular challenge (10). For example, rates for baseline data of $47 \%$ of eligible clients in a fiscal year have been cited (14). In a longitudinal study of multiple treatment programs, Harrison and Asche (54) obtained eligible baseline data from $61 \%$ of targeted substance misuse treatment programs. Future projects may increase their baseline eligibility levels by establishing the routine use of outcomes measures at an organisational level before, rather than simultaneously, with the implementation of follow-up procedures. In addition, our sample has significantly higher comorbidity rates (64\% to $71 \%)$ than previously reported research (55) and may represent a relatively complex clinical population.

This research has demonstrated that the implementation of telephone follow-up procedures can return adequate follow-up data at a reasonable cost. However, the study also highlights some of the difficulties inherent in this process. Ultimately, the implementation of outcome measures across multiple treatment settings concurrently with telephone follow-up protocols represents a significant challenge for both researchers and treatment providers. The results of this study suggest that it can be feasible but, as suggested previously (10), the demands are onerous. Our results suggest that a phased implementation of routine intake and follow-up outcome measures may be more appropriate than implementing the two procedures simultaneously. Within this, follow-up response may be increased by exploring strategies for supporting ethnic minorities and exploring personality and motivational variables. 


\section{REFERENCES}

1. Horsfall J, Cleary M, Hunt GE. Developing Partnerships in Mental Health to Bridge the Research-Practitioner Gap. Perspectives in Psychiatric Care. 2011;47:6-12.

2. Rosenheck RA, Seibyl CL. A longitudinal perspective on monitoring outcomes of an innovative program. Psychiatric Services. 2005;56:301-307.

3. Proctor EK, Landsverk J, Aarons G, Chambers D, Glisson C, Mittman B. Implementation research in mental health services: an emerging science with conceptual, methodological, and training challenges. Administration and Policy in Mental Health. 2009;36:24-34.

4. Tansella M, Thornicroft G. Implementation science: understanding the translation of evidence into practice. . The British Journal of Psychiatry. 2009;195:283-285.

5. Mendel P, Meredith LS, Schoenbaum M, Sherbourne CD, Wells KB. Interventions in organisational and community context: A framework for building evidence on dissemination and implementation in health services research. . Administration and Policy in Mental Health. 2008;35:21-37.

6. Bootsmiller BJ, Ribisl KM, Mowbray CT, Davidson WS, Walton MA, Herman SE. Methods of ensuring high follow-up rates: Lessons from a longitudinal study of dual diagnosed participants. Substance Use and Misuse. 1998;33:2665-2685.

7. Oudejans SCC, Schippers GM, Merkx MJM, Schramade MH, Koeter MWJ, van den Brink W. Feasibility and validity of low-budget telephonic follow-up interviews in routine outcome monitoring of substance abuse treatment. Addiction. 2009;104:11381146.

8. Walton MA, Ramanathan CS, Reischl TM. Tracking substance abusers in longitudinal research: Understanding follow-up contact difficulty. American Journal of Community Psychology. 1998;26:233-253. 
9. Gerstein DR, Johnson RA. Nonresponse and selection bias in treatment follow-up studies. Substance Use Misuse. 2000;35:971-1014.

10. Tiet QQ, Byrnes HF, Barnett P, Finney JW. A practical system for monitoring the outcomes of substance use disorder patients. . Journal of Substance Abuse Treatment,. 2006;30:337-347.

11. Corsi KF, Van Hunnik B, Kwiatowski CF, Booth RE. Computerized tracking and follow-up techniques in longitudinal research with drug users. . Health Services and Outcomes Research Methodology. 2006;6:101-110.

12. Moos RH, Finney JW, Suchinsky R. Outcomes monitoring for patients with substance use disorders: IV. Cohort 2 patients' 6-12 month treatment outcomes. Palo Alto, CA: Program Evaluation and Resource Center and HSR\&D Center for Health Care Evaluation.;2000.

13. Otilingram PG, Ritsher JB, Finney JW, Moos RH, Suchinsky R. Outcomes monitoring for patients with substance use disorders: $V$. Cohort 3 patients' characteristics, treatment, and treatment outcomes. Palo Alto, CA: Program Evaluation and Resource Center and HSR\&D Center for Health Care Evaluation.;2002.

14. Moos RH, Finderman B, Finney JW, Suchinsky R. Outcomes monitoring for patients with substance use disorders: III. Cohort 2 patients' characteristics and treatment at baseline. Palo Alto, CA: Program Evaluation and Resource Center and HSR\&D Center for Health Care Evaluation.;1999.

15. Evans E, Hser YI. Pilot-testing a statewide outcome monitoring system: overview of the California Treatment Outcome Project (CALTOP). Journal of Psychoactive Drugs. 2004;2 (suppl):109-114. 
16. Rohrer JE, Vaughan MS, Cadoret R, Zwick J. Follow-up contact bias in evaluation of substance abuse treatment programs. Administration and Policy in Mental Health. 1999;26:207-212.

17. Flynn PM, Simpson DD, Anglin MD, Hubbard RL. Comment on 'nonresponse and selection bias in treatment follow-up studies'. Substance Use Misuse. 2001;36:17531757.

18. McLellan AT, Kushner H, Metzger D, Peters R, Smith I, Grissom G. The fifth edition of the Addiction Severity Index [Electronic Edition]. . Journal of Substance Abuse Treatment. 1995;9:199-213.

19. McKenzie M, Peterson Tulsky J, Long HJ, Chesney M. Tracking and follow-up of marginalised populations: A review. Journal of Health Care for the Poor and Underserved. 1999;10:409-429.

20. Gordon R, Heim D, MacAskill S. Rethinking drinking cultures: A review of drinking cultures and a reconstructed dimensional approach. Public Health. 2012;126:3-11.

21. Ståhlbrandt H, Andersson C, Johnsson KO, Tollison SJ, Berglund M, Larimer ME. Cross-Cultural Patterns in College Student Drinking and its Consequences-A Comparison between the USA and Sweden. Alcohol \& Alcoholism. 2006;41:i3-i7.

22. Vederhus J, Laudet A, Kristensen $\varnothing$, Clausen T. Obstacles to 12-step group participation as seen by addiction professionals: Comparing Norway to the United States. Journal of Substance Abuse Treatment. 2010;39:210-217.

23. Lundberg I, Thakker KD, Hällström T, Forsell Y. Determinants of non-participation, and the effects of non-participation on potential cause-effect relationships, in the PART study on mental disorders. Soc Psychiatry Psychiatr Epidemiol. 2005;40:475483. 
24. Cunradi CB, Moore R, Killoran M, Ames G. Survey nonresponse bias among young adults: the role of alcohol, tobacco, and drugs. Substance Use \& Misuse. 2005;40:171185.

25. Eckers RM. 'Cultural fraud': the role of culture in drug abuse. Drug and Alcohol Review. 2005;24:157-163.

26. Lawrinson P, Copeland J, Indig D. Development and validation of a brief instrument for routine outcome monitoring in opioid maintenance pharmacotherapy services: The brief treatment outcome measure (BTOM). . Drug and Alcohol Dependence. 2005;80:125-133.

27. Alterman AI, Cacciola JS, Habing B, Lynch KG. Addiction severity index recent and lifetime summary indexes based on nonparametric item response theory methods. Psychological Assessment. 2007;19:119-132.

28. Cacciola JS, Pecoraro A, Alterman AI. Development of ASI psychiatric severity cutoff scores to identify co-occurring psychiatric disorders. . International Journal of Mental Health and Addiction. 2008;6:77-92.

29. Currie SR, el-Guebaly N, Coulson R, Hodgins D, Mansley C. Factor validation of the addiction severity index scale structure in persons with concurrent disorders. Psychological Assessment. 2004;16:326-329.

30. Enstrom C. Social workers ability to assess how clients experience investigation sessions- with and without the ASI. Journal of Social Work. 2010;9:309-321.

31. Griffin ML, Kolodziej ME, Weiss RD. Measuring principle substance of abuse in comorbid patients for clinical research. Addictive Behaviours. 2009;34:826-829.

32. Reelick NF, Wierdsma AI. The addiction severity index as a predictor of the use of mental health care. Psychology of Addictive Behaviours. 2006;20:214-218. 
33. Lovibond PF, Lovibond SH. The structure of negative emotional states: Comparison of the Depression Anxiety Stress Scale (DASS) with the Beck depression and anxiety the inventories. . Behavior Research and Therapy. 1995;33:335-343.

34. Sklar SM, Annis HM, Turner NE. Group comparisons of coping self-efficacy between alcohol and cocaine abusers seeking treatment. Psychology of Addictive Behaviors. 1999;13:123-133.

35. Clark D. Craving for alcohol. Journal of Psychopharmacology. 1994;9:73.

36. Corrigan PW, Salver M, Ralph RO, Songster Y, Keck L. Examining the factor structure of the Recovery Assessment Scale. Schizophrenia Bulletin. 2004;30:10351041.

37. Scheier MF, Wrosch C, Baum A, et al. The life engagement test: Assessing purpose in life. Journal of Behavioral Medicine. 2006;29:291-298.

38. Keyes CLM, Wissing M, Potgieter JP, Temane M, Kruger A, van Rooy S. Evaluation of the mental health continuum-short form (MHC-SF) in Setswana-speaking South Africans. Clinical Psychology \& Psychotherapy. 2008;15:181-192.

39. De Leon G. Therapeutic communities for addictions: A theoretical framework. The International Journal of the Addictions. 1995;30:1603-1645.

40. Schaler JA. Spiritual thinking in addiction-treatment providers: The Spiritual Belief Scale (SBS). Alcoholism Treatment Quarterly. 1996;14:7-33.

41. Connors GJ, Tonigan JS, Miller WR. A measure of religious background and behavior for use in behavior change research. Psychology of Addictive Behaviors. 1996;10:90-96.

42. Underwood LG, Teresi JA. The Daily Spiritual Experience Scale: Development, theoretical description, reliability, exploratory factor analysis, and preliminary 
construct validity using health-related data. Annals of Behavioral Medicine. 2002;24:22-33.

43. Thompson LY, R. SC, Michael ST, et al. Dispositional Forgiveness of Self, Others, and Situations. Journal of Personality and Social Psychology. 2005;73:313-360.

44. Thompson LY, Snyder CR. Measuring forgiveness. In: Lopez SJ, Snyder CR, eds. Positive psychological assessment: A handbook of models and measures. Washington, DC: American Psychological Association; 2003:301 - 312.

45. Buss AH, Perry M. The Aggression Questionnaire. Journal of Personality and Social Psychology. 1992;63:452-459.

46. Ribisl KM, Walton MA, Mowbray CT, Luke DA, Davidson II WS, Bootsmiller BJ. Minimizing participant attrition in panel studies through the use of effective retention and tracking strategies: Review and recommendations. Evaluation and Program Planning. 1996;19:1-25.

47. Kertesz SG, Crouch K, Milby J, Cusimano RA, Schumacher J. Housing First for Homeless Persons with Active Addiction: Are We Overreaching? . Milbank Quarterly. 2009;87:495-534.

48. Brunette MF, Mueser KT, Drake RE. A review of research on residential programs for people with severe mental illness and co-occurring substance use disorders. Drug and Alcohol Review. 2004;23:471-481.

49. Passetti LL, Godley SH, Scott CK, Siekmann M. A low-cost follow-up resource: Using the world wide web to maximise client location efforts. American Journal of Evaluation. 2000;21:195-203.

50. Mäkelä K. Studies of the reliability and validity of the Addiction Severity Index. Addiction. 2004;99:398-410. 
51. Secades-Villa R, Fernandez-Hermida JR. The validity of self-reports in a follow-up study with drug addicts. Addictive Behaviors. 2003;28:1175-1182.

52. Australian Bureau of Statistics \& Australian Institute of Health and Welfare. The Health and Welfare of Australia's Aboriginal and Torres Strait Islander Peoples 2005. In: Welfare ABoSAIoHa, ed. Canberra2005.

53. Marcus B, Schutz A. Who are the people reluctant to participate in research? Personality correlates of four different types of nonresponse as inferred from self- and observer ratings. Journal of Personality. 2005;73:959-984.

54. Harrison PA, Asche SE. Outcomes monitoring in Minnesota: Treatment implications, practical limitations. . Journal of Substance Abuse Treatment. 2001;11:173-183.

55. Mortlock KS, Deane FP, Crowe TP. Screening for mental disorder comorbidity in Australian alcohol and other drug residential treatment settings. . Journal of Substance Abuse Treatment. 2011;40:397-404. 


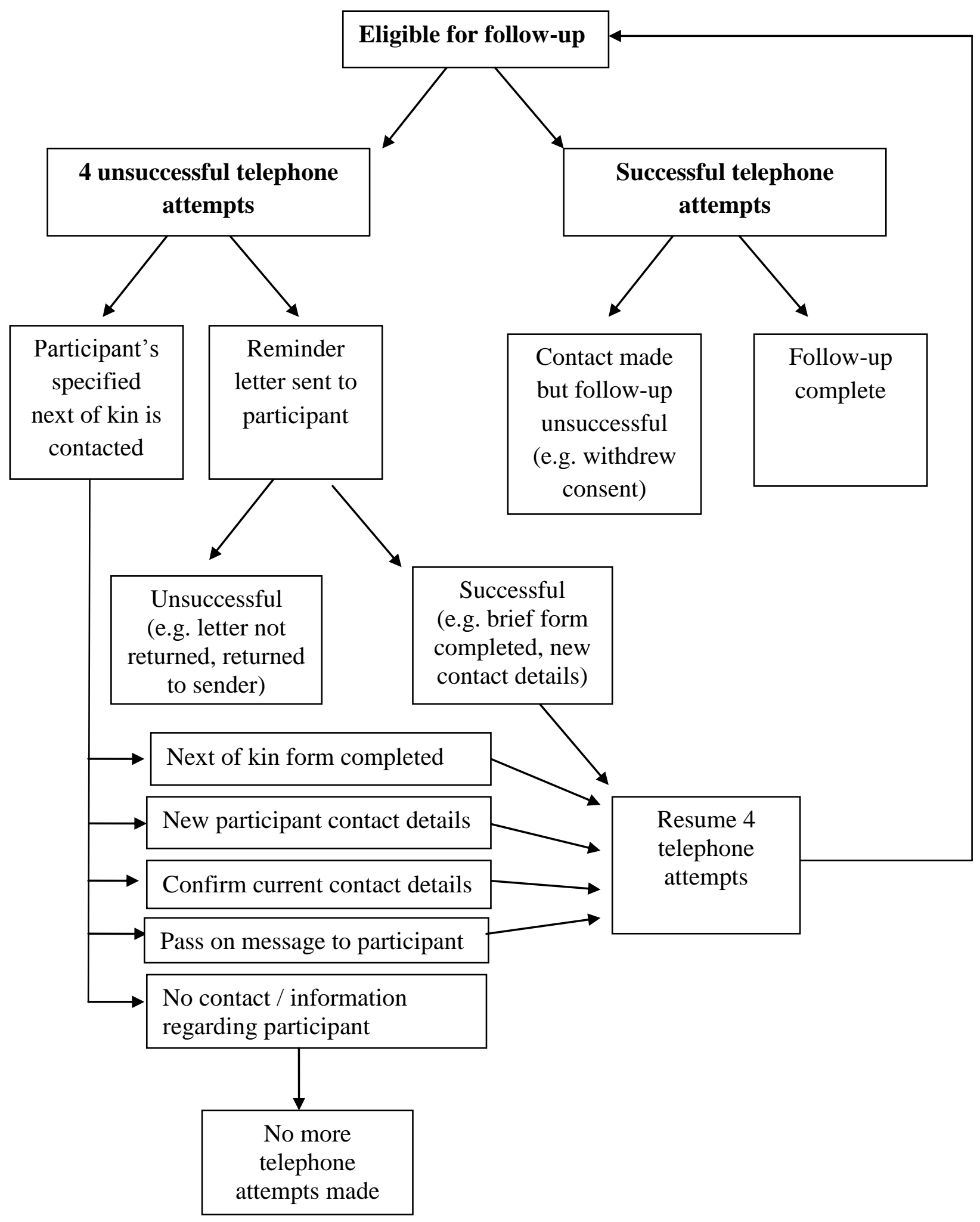

Figure 1: Telephone follow-up procedure 


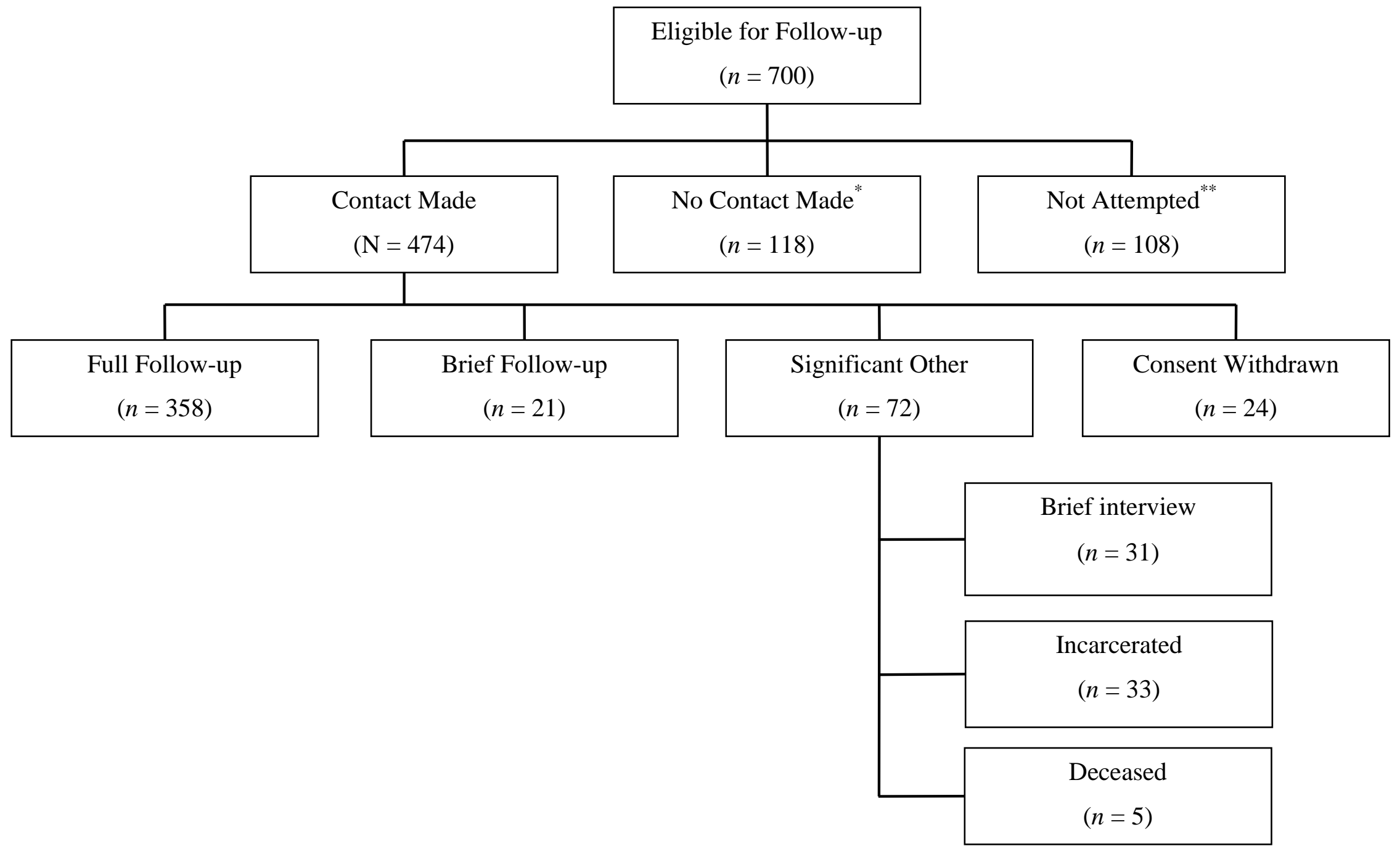

Figure 2. Breakdown of follow-up responses

* includes participants who were unable to be contacted despite repeated attempts

** includes participants who were not targeted for follow-up due to service and/or research level administrative errors (e.g. not detected in weekly data downloads) 
Table 1: Demographics According to Group

\begin{tabular}{|c|c|c|c|}
\hline Demographics & $\begin{array}{c}\text { No Attempt } \\
\text { Made ( } n=108)\end{array}$ & $\begin{array}{l}\text { Contacted } \\
(n=474)\end{array}$ & $\begin{array}{c}\text { No Contact } \\
\text { Made }(n=118)\end{array}$ \\
\hline Age $(M$ years, $S D)$ & $35.24(9.69)$ & $36.12(10.81)$ & $34.52(10.42)$ \\
\hline \multicolumn{4}{|l|}{ Gender $(\%)$} \\
\hline Male & 80.4 & 82.3 & 89 \\
\hline Female & 19.6 & 17.3 & 11 \\
\hline \multicolumn{4}{|l|}{ Marital status (\%) } \\
\hline Single / never married & 66.5 & 60.5 & 67.8 \\
\hline Divorced / separated & 21.8 & 26.6 & 18.7 \\
\hline Married & 6.2 & 6.5 & 7.6 \\
\hline Non-specified & 5.5 & 6.4 & 5.9 \\
\hline \multicolumn{4}{|l|}{ Ethnicity (\%) } \\
\hline Anglo Australian & 69.5 & 77.2 & 65.3 \\
\hline $\operatorname{ATSI}^{1}$ & 14.0 & 7.8 & 12.7 \\
\hline Other $^{2}$ & 12.1 & 15 & 22 \\
\hline \multicolumn{4}{|l|}{ Religion (\%) } \\
\hline Christian & 64.6 & 64.4 & 55.1 \\
\hline Nil & 20.2 & 24.3 & 32.2 \\
\hline Other ${ }^{3}$ & 15.2 & 11.3 & 12.7 \\
\hline \multicolumn{4}{|l|}{ Highest Education (\%) } \\
\hline Primary & .8 & 1.7 & 2.5 \\
\hline Lower secondary & 26.8 & 29.3 & 36.4 \\
\hline Upper secondary & 61.5 & 56.3 & 51.7 \\
\hline
\end{tabular}


Post-secondary ${ }^{4}$

Employment status in previous 3 years

$(\%)$

Full-time work

Part-time work

Unemployed

Controlled environment

Other $^{5}$

Primary Substance

Alcohol

Cannabis

Other drugs 6

Years abusing primary substance

$(M, S D)$

Number of previous treatments $(M, S D)$

Days in treatment $(M, S D)$
10.9

12.7

9.4

27.6

28.5

23.7

26.1

23.8

28.9

26.1

23.4

24.6

5.4

8.4

9.3

14.8

15.9

13.5
54.5

13.9

31.6

30.0

33.9

$13.41(10.25) \quad 18.35(10.13) \quad 15.65(10.21)$

56.8

9.3

14.3 
Table 2: Intake Addiction Severity Index Composite Score according to contact groups

\begin{tabular}{lccc}
\hline \multicolumn{1}{c}{ Intake Addiction } & No Attempt Made & Contacted & No Contact Made \\
Severity Index & $M(S D)$ & $M(S D)$ & $M(S D)$ \\
Composite Score & $.27(.29)$ & $.27(.33)$ & $.27(.32)$ \\
\hline Medical & $.76(.20)$ & $.77(.20)$ & $.80(.19)$ \\
Employment & $.43(.36)$ & $.48(.35)$ & $.46(.36)$ \\
Alcohol & $.14(.13)$ & $.14(.12)$ & $.11(.11)$ \\
Drug & $.12(.06)$ & $.30(.26)$ & $.33(.20)$ \\
Legal & $.28(.24)$ & $.48(.25)$ & $.43(.28)$ \\
Psychiatric & & & \\
\hline
\end{tabular}

